\title{
HOCHSCHILD HOMOLOGY IN A BRAIDED TENSOR CATEGORY
}

\author{
JOHN C. BAEZ
}

\begin{abstract}
An $r$-algebra is an algebra $A$ over $k$ equipped with a Yang-Baxter operator $R: A \otimes A \rightarrow A \otimes A$ such that $R(1 \otimes a)=a \otimes 1, R(a \otimes 1)=1 \otimes a$, and the quasitriangularity conditions $R(m \otimes I)=(I \otimes m)(R \otimes I)(I \otimes R)$ and $R(I \otimes m)=(m \otimes I)(I \otimes R)(R \otimes I)$ hold, where $m: A \otimes A \rightarrow A$ is the multiplication map and $I: A \rightarrow A$ is the identity. $R$-algebras arise naturally as algebra objects in a braided tensor category of $k$-modules (e.g., the category of representations of a quantum group). If $m=m R^{2}$, then $A$ is both a left and right module over the braided tensor product $A^{e}=A \widehat{\otimes} A^{\text {op }}$, where $A^{\text {op }}$ is simply $A$ equipped with the "opposite" multiplication map $m^{\text {op }}=m R$. Moreover, there is an explicit chain complex computing the braided Hochschild homology $H^{R}(A)=\operatorname{Tor}^{A^{e}}(A, A)$. When $m=m R$ and $R^{2}=\mathrm{id}_{A \otimes A}$, this chain complex admits a generalized shuffle product, and there is a homomorphism from the $r$-commutative differential forms $\Omega_{R}(A)$ to $H^{R}(A)$.
\end{abstract}

\section{INTRODUCTION}

It has been known for some time that many constructions in commutative algebra can be profitably extended to "supercommutative" algebras, that is, $\mathbf{Z}_{2}$ graded algebras satisfying $a b=(-1)^{\operatorname{deg} a \operatorname{deg} b} b a$. To do so, one follows the simple rule that the twist map

$$
a \otimes b \mapsto b \otimes a
$$

should be replaced everywhere by the graded twist map

$$
a \otimes b \mapsto(-1)^{\operatorname{deg} a \operatorname{deg} b} b \otimes a .
$$

This rule appears frequently in physics, where even and odd variables are used to describe bosonic and fermionic degrees of freedom, respectively.

More recently, developments in mathematical physics have led to a further generalization of commutativity, in which the role of the symmetric group is taken instead by the braid group. The twist map is thus replaced by an arbitrary Yang-Baxter operator

$$
a \otimes b \mapsto R(a \otimes b)
$$

satisfying certain compatibility relations with multiplication. More precisely, suppose that $A$ is a unital algebra over a commutative ring $k$, and let $m: A \otimes$ $A \rightarrow A$ denote the multiplication map and $I: A \rightarrow A$ the identity map. Then

Received by the editors January 30, 1992, and, in revised form, October 6, 1993.

1991 Mathematics Subject Classification. Primary 58B30; Secondary 16W30, 18 D10. 
$A$ is an $r$-algebra if there is an $r$-structure $R: A \otimes A \rightarrow A \otimes A$, an invertible linear map satisfying: (1) the Yang-Baxter equation:

$$
(R \otimes I)(I \otimes R)(R \otimes I)=(I \otimes R)(R \otimes I)(I \otimes R),
$$

(2) the quasitriangularity conditions:

$$
R(m \otimes I)=(I \otimes m)(R \otimes I)(I \otimes R), \quad R(I \otimes m)=(m \otimes I)(I \otimes R)(R \otimes I),
$$

and (3) the following conditions for all $a \in A$ :

$$
R(1 \otimes a)=a \otimes 1, \quad R(a \otimes 1)=1 \otimes a .
$$

We say that $A$ is " $r$-commutative" if in addition $m=m R$. For example, every $\mathbf{Z}_{2}$-graded algebra is an $r$-algebra with

$$
R(a \otimes b)=(-1)^{\operatorname{deg} a \operatorname{deg} b} b \otimes a,
$$

and then $r$-commutativity reduces to supercommutativity. Many other interesting noncommutative algebras are also $r$-commutative. These include quantum groups [6,7], quantum matrix algebras [7], quantum vector spaces [20, 22, 23, 31,33], noncommutative tori [4, 26], the Weyl and Clifford algebras, and certain universal enveloping algebras. $R$-commutative algebras also arise quite generally as semiclassical limits in the quantization of Poisson algebras.

As noted by Manin [20,21], $r$-algebras may be defined simply as algebra objects in braided tensor categories of $k$-modules. Roughly speaking, a braided tensor category is a category with tensor products such that for any objects $U, V$ there is a "braiding"

$$
R_{U, V}: U \otimes V \rightarrow V \otimes U,
$$

an isomorphism satisfying axioms generalizing the properties of the twist map. The concept of a braided tensor category has its roots in Mac Lane's work [18] on the category-theoretic foundations of associativity and commutativity, and a certain class, the Tannakian categories, have long found wide application in number theory and other subjects [5, 27]. More recently, braided tensor categories have been seen to arise naturally in a variety of closely related situations. First, given a $k$-module $V$ and an isomorphism $R: V \otimes V \rightarrow V \otimes V$ satisfying the Yang-Baxter equation, one may canonically construct a braided tensor category of $k$-modules containing $V$ for which $R_{V, V}=R$ [17]. Second, the category of representations of a quantum group over $k$ is a braided tensor category of $k$-modules, with the universal $R$-matrix serving to define the braiding $[19,30]$. Third, certain braided tensor categories give rise to invariants of knots and three-manifolds $[10,25]$. Fourth, quantum field theories give rise to braided tensor categories of vector spaces over $\mathbf{C}$ [9]. These field theories, in turn, are closely related to statistical mechanics models [32].

Manin has proposed an approach to noncommutative geometry in which one generalizes standard constructions of commutative algebra to algebras in a braided tensor category. The author has taken this up [1], studying an analog of differential forms that is applicable to any "strong" $r$-commutative algebra, that is, one with $R^{2}=\mathrm{id}_{A \otimes A}$. Certain nonstrong cases are also understood [2]. This approach to noncommutative geometry may at first seem orthogonal to that taken by Connes [4], Tsygan [29], Loday-Quillen [24] and others, in which one works with cyclic Hochschild chains to define cyclic homology as an analog 
of de Rham cohomology for noncommutative algebras. Here, however, we begin a unification of these approaches by defining a generalization of Hochshild homology for algebras in a braided tensor category. This extends the previous generalization of Hochschild homology to supercommutative algebras.

The general strategy is to systematically replace the twist map wherever it appears by the appropriate braiding. We begin by associating to any $r$-algebra $A$ an "opposite" $r$-algebra $A^{\text {op }}$ with multiplication map $m^{\text {op }}=m R$. The algebra $A$ then becomes a left module over $A^{e}=A \widehat{\otimes} A^{\text {op }}$, where $\widehat{\otimes}$ denotes the "braided tensor product." We construct a flat resolution of $A$ as an $A^{e}$ module when $A$ is flat over $k$. This enables us to compute $\operatorname{Tor}^{A^{e}}(E, A)$ for a right $A^{e}$-module $E$ by means of an explicit chain complex. The algebra $A$ itself is a right $A^{e}$-module when $A$ is "weakly $r$-commutative," that is, $m=m R^{2}$. This allows the construction of the "braided Hochschild homology" $H^{R}(A)=\operatorname{Tor}^{A^{e}}(A, A)$. In particular, when $A$ is strong and $r$-commutative, there is a product on $H^{R}(A)$ induced by a generalized shuffle product on the corresponding chain complex. Recall that in the commutative case there is a homomorphism from the differential forms over $A$ to the Hochschild homology of $A$, which is an isomorphism when $A$ is smooth [11]. Here we construct a homomorphism from the $r$-commutative generalization of differential forms to $H^{R}(A)$.

The author is indebted to Ezra Getzler for raising the idea of generalizing Hochschild cohomology to algebras in braided tensor categories, and for pointing out the difficulties. The author would also like to thank Minhyong Kim for many helpful conversations.

\section{Algebras IN BRAIDED TENSOR CATEGORIES}

In earlier work on $r$-commutative algebra [1,2], we worked directly from the definition given above, avoiding the introduction of category theory. In homological considerations, however, a small investment in the language of tensor categories is amply repaid.

A monoidal category is a category $\mathscr{C}$ equipped with a bifunctor $\otimes: \mathscr{C} \times \mathscr{C} \rightarrow$ $\mathscr{C}$, a functorial isomorphism

$$
A_{U, V, W}: U \otimes(V \otimes W) \rightarrow(U \otimes V) \otimes W
$$

called the associativity constraint, and an object 1 equipped with functorial isomorphisms

$$
l_{V}: 1 \otimes V \rightarrow V, \quad r_{V}: V \otimes 1 \rightarrow V,
$$

satisfying:

(1) the pentagon axiom, namely that all diagrams

$$
\begin{array}{cc}
U \otimes(V \otimes(W \otimes X)) \stackrel{A}{\longrightarrow}(U \otimes V) \otimes(W \otimes X) \stackrel{A}{\longrightarrow}(U \otimes(V \otimes W)) \otimes X \\
\quad \text { id } \otimes A \downarrow & \uparrow A \otimes \text { id } \\
U \otimes((V \otimes W) \otimes X) \longrightarrow & (U \otimes(V \otimes W)) \otimes X
\end{array}
$$

commute, and 
(2) The triangle axiom, namely that all diagrams

$$
\begin{array}{cc}
(U \otimes 1) \otimes V \stackrel{A}{\longrightarrow} U \otimes(1 \otimes V) \\
r \otimes \text { id } \searrow & \swarrow \mathrm{id} \otimes l
\end{array}
$$

$$
U \otimes V
$$

commute.

A monoidal category $\mathscr{C}$ is said to be braided if for all objects $U, V$ there exist functorial isomorphisms

$$
R_{U, V}: U \otimes V \rightarrow V \otimes U,
$$

satisfying the hexagon axioms, namely that all diagrams

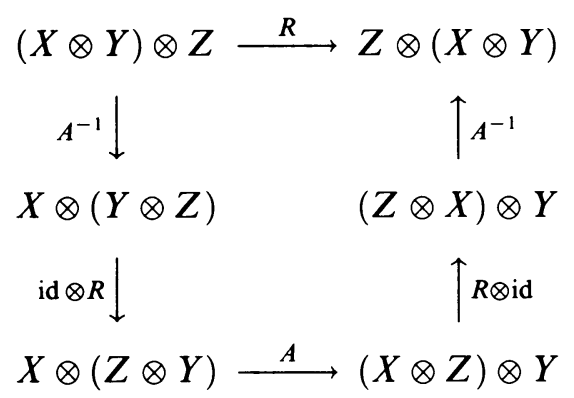

and

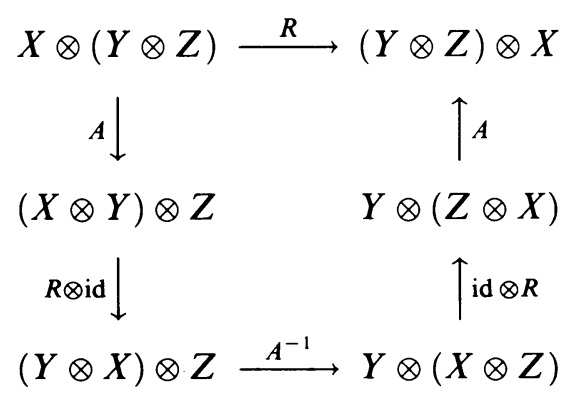

commute. If $\mathscr{C}$ is a braided monoidal category such that $R_{V, U} \circ R_{U, V}=$ $\operatorname{id}_{U \otimes V}$ for all objects $U, V$, we say $\mathscr{C}$ is a symmetric monoidal category. In a symmetric monoidal category, either of the two hexagon axioms implies the other.

Given a commutative ring $k$, we define a tensor category of $k$-modules to be a monoidal category $\mathscr{C}$ equipped with a faithful functor $F$ to the category of $k$-modules such that: (1) $F(U \otimes V)=F(U) \otimes F(V),(2) F\left(A_{U, V, W}\right)$ is the natural isomorphism of $k$-modules,

$$
F(U) \otimes(F(V) \otimes F(W)) \stackrel{\sim}{\rightarrow}(F(U) \otimes F(V)) \otimes F(W),
$$

(3) $F(1)=k$, (4) $F\left(l_{V}\right)$ and $F\left(r_{V}\right)$ are the natural isomorphisms of $k$ modules,

$$
k \otimes F(V) \stackrel{\sim}{\rightarrow} F(V), \quad F(V) \otimes k \stackrel{\sim}{\rightarrow} F(V) .
$$

A braided (resp. symmetric) tensor category of $k$-modules is a braided (resp. symmetric) monoidal category equipped with a faithful functor $F$ to the category of $k$-modules such that properties (1)-(5) above hold. The point is that 
while the associativity constraint $A$ in $\mathscr{C}$ is mapped to the usual associativity constraint for $k$-modules, this need not hold of the braiding $R$. It is also important that while distinct objects in $\mathscr{C}$ may correspond to the same $k$-module, distinct morphisms in $\mathscr{C}$ correspond to distinct $k$-module morphisms.

Henceforth we shall work in a fixed braided tensor category $\mathscr{V}$ of $k$-modules, where $k$ is some commutative ring. Because the associativity constraint in $\mathscr{V}$ maps to the standard one in the category of $k$-modules, and the pentagon axiom allows us to reparenthesize tensor products in $\mathscr{V}$ in a coherent manner, we simply omit parentheses around tensor products in $\mathscr{V}$, and omit all mention of the associativity constraint. We also identify objects and morphisms in $\mathscr{V}$ with their images under $F$ when this will cause no confusion.

One can easily prove generalized forms of the Yang-Baxter equation and the quasitriangularity conditions in a braided tensor category. The quasitriangularity conditions state that, for any objects $U, V, W, X$ in $\mathscr{V}$ and morphism $f: U \otimes V \rightarrow X$, the following diagrams commute:

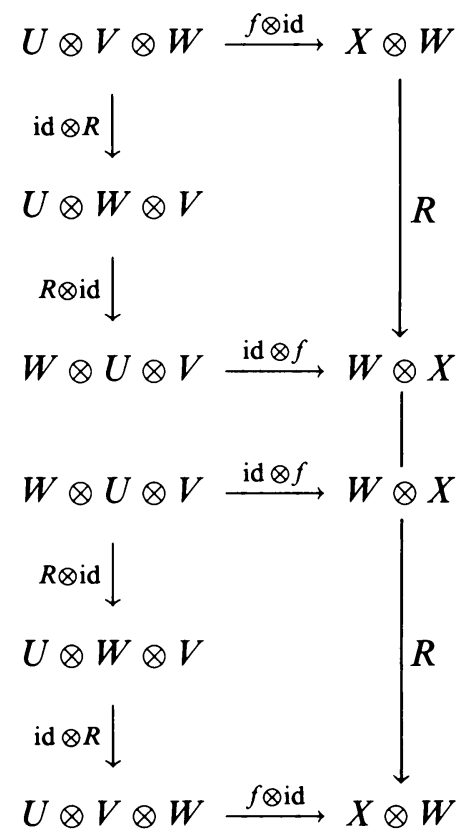

These conditions follow from hexagon axiom together with the functoriality of the braiding. There is also a dual version for morphisms $f: X \rightarrow U \otimes V$, which appears in the definition of a quasitriangular Hopf algebra, but we will not need this here. The Yang-Baxter equation follows from the first quasitriangularity condition upon taking $X=V \otimes U, f=R_{U, V}$. It states that, for any objects $U, V, W$ in $\mathscr{V}$, the following diagram commutes:

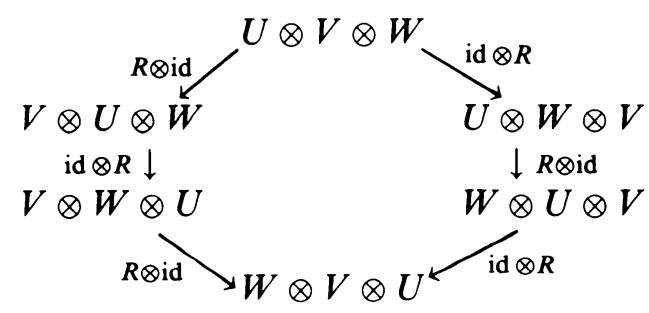




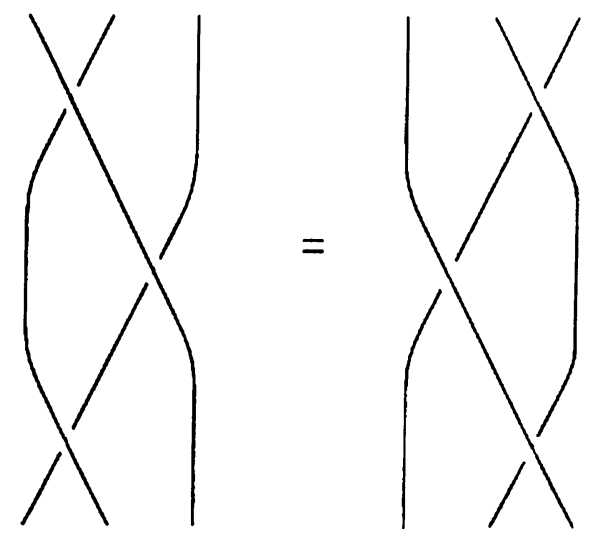

FIGURE 1. The Yang-Baxter equation
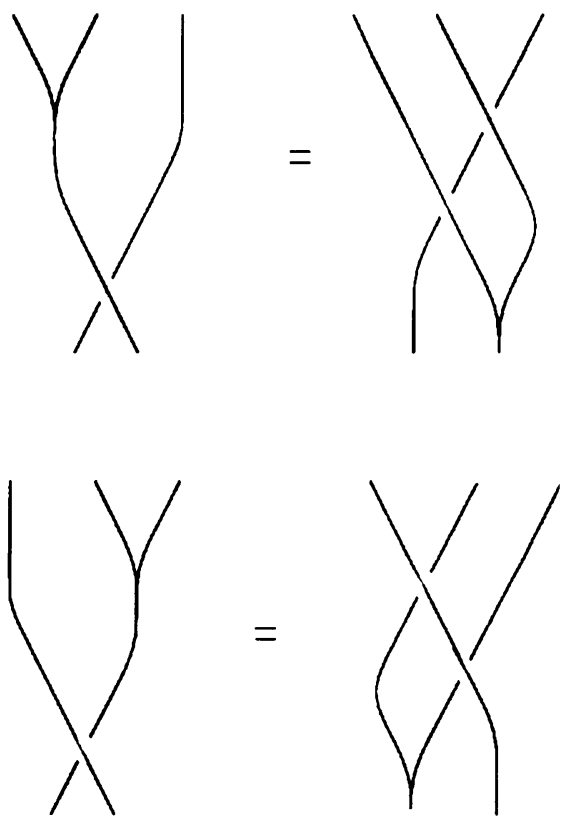

FIGURE 2. The quasitriangularity conditions

Using quasitriangularity for the morphism $l: k \otimes k \rightarrow k$ and property (4) of tensor categories of $k$-modules, it follows that

$$
R_{k, V}(x \otimes v)=v \otimes x
$$

and

$$
R_{V, k}(v \otimes x)=x \otimes v
$$

for all $x \in k$ and $v \in V$. From these it also follows that

$$
l_{V} R_{V, k}=r_{V}, \quad r_{V} R_{k, V}=l_{V} .
$$

It proves very convenient to compute in a braided tensor category using pictures. Calculations of this sort have long been used in knot theory, especially by Kauffman $[15,16]$. Their category-theoretic foundations have recently been explored by Joyal and Street $[12,13]$. If we denote the braiding 

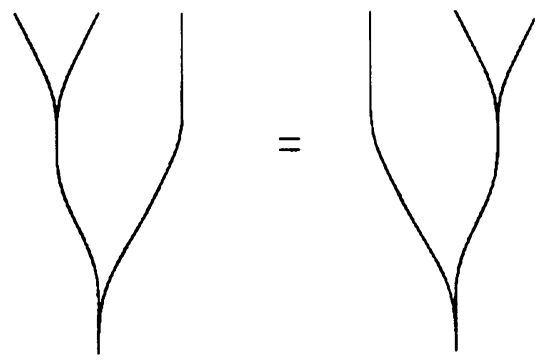

FIGURE 3. Associativity

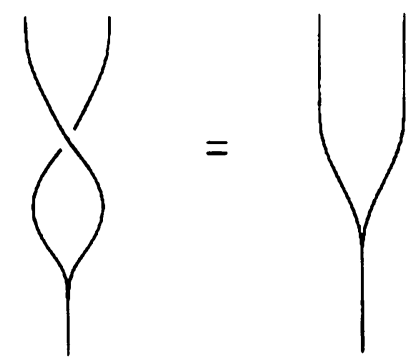

FIGURE 4. $R$-commutativity

$R_{U, V}: U \otimes V \rightarrow V \otimes U$ by the crossing of two strands and denote identity morphisms by a vertical line, then the Yang-Baxter equation may be diagrammed as in Figure 1. If in addition we denote the morphism $f: U \otimes V \rightarrow W$ by the joining of two strands, the quasitriangularity conditions may be diagrammed as in Figure 2.

Now let us turn to the study of algebras in a braided tensor category. We will only develop the ingredients necessary to set up Hochschild homology. To begin with, we say that $A$ is an algebra in $\mathscr{V}$ if $A$ is an object in $\mathscr{V}$ equipped with a multiplication map $m \in \operatorname{Hom}_{\mathscr{V}}(V \otimes V, V)$ satisfying associativity:

$$
m\left(m \otimes \mathrm{id}_{A}\right)=m\left(\mathrm{id}_{A} \otimes m\right)
$$

and a unit map $l \in \operatorname{Hom}_{\mathscr{V}}(k, V)$ satisfying

$$
m\left(l \otimes \mathrm{id}_{A}\right)=l_{A}, \quad m\left(\mathrm{id}_{A} \otimes l\right)=r_{A} .
$$

It is easy to check that an algebra in $\mathscr{V}$ is indeed an $r$-algebra as defined in the introduction, taking $R=R_{A, A}$. Conversely, given an $r$-algebra over a commutative ring $k$, we may regard it as an algebra in some braided tensor category of $k$-modules, using a construction of Lyubashenko [17].

We say that an algebra $A$ in $\mathscr{V}$ is $r$-commutative if $m=m R_{A, A}$. Using the joining of two strands to denote the multiplication map, associativity corresponds to Figure 3, while $r$-commutativity corresponds to Figure 4. Even when $A$ is not $r$-commutative, $m R_{A, A}$ is an associative product on $A$. In fact:

Lemma 1. Let $A$ be an algebra in $V$. Let $A^{\text {op }}$ denote $A$ with the multiplication map $m^{\mathrm{op}}=m R_{A, A}$ and the same unit map $l$. Then $A^{\mathrm{op}}$ is an algebra in $\mathscr{V}$.

Proof. First we check associativity, by a four-step calculation shown in Figure 5. Writing $R=R_{A, A}$ and $\mathrm{id}_{A}=I$, by definition we have $m^{\mathrm{op}}\left(m^{\mathrm{op}} \otimes I\right)=$ $m R(m \otimes I)(R \otimes I)$. 


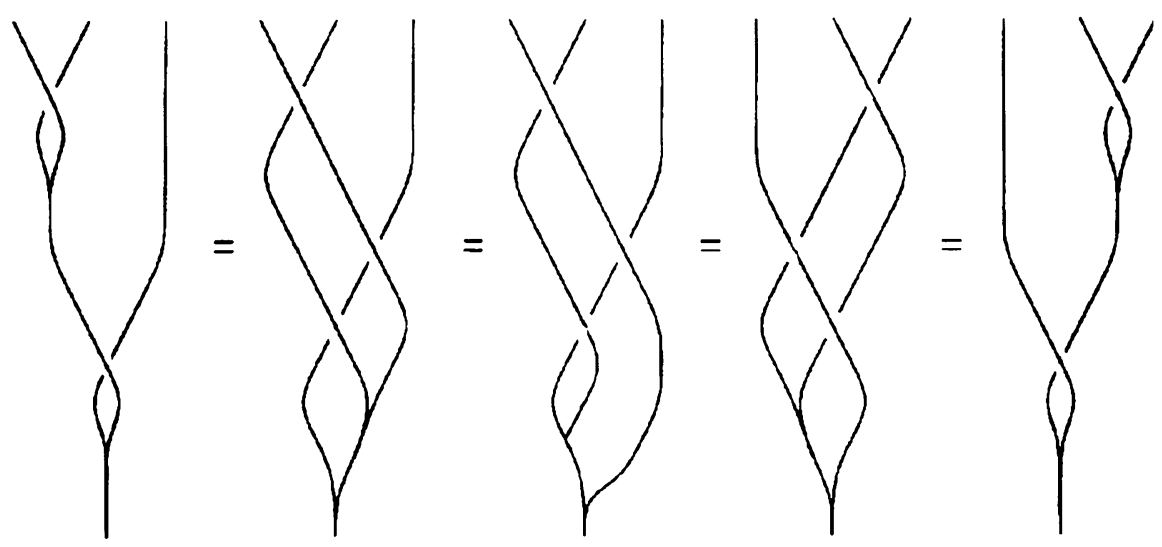

FIGURE 5. Associativity of the opposite algebra

Step 1: By quasitriangularity the latter equals

$$
m(I \otimes m)(R \otimes I)(I \otimes R)(R \otimes I) .
$$

Step 2: By associativity this equals

$$
m(m \otimes I)(R \otimes I)(I \otimes R)(R \otimes I) .
$$

Step 3: By the Yang-Baxter equation this equals

$$
m(m \otimes I)(I \otimes R)(R \otimes I)(I \otimes R) .
$$

Step 4: By quasitriangularity this equals

$$
m R(I \otimes m)(I \otimes R),
$$

which equals $m^{\mathrm{op}}\left(I \otimes m^{\mathrm{op}}\right)$, as desired. Checking that $l$ is a unit map for $m^{\mathrm{op}}$ is straightforward:

$$
m^{\mathrm{op}}(\imath \otimes I)=m(I \otimes l) R_{k, A}=r_{A} R_{k, A}=l_{A},
$$

and

$$
m^{\mathrm{op}}(I \otimes \imath)=m(\imath \otimes I) R_{A, k}=l_{A} R_{A, k}=r_{A} .
$$

The $r$-algebra $A^{\text {op }}$ is called the opposite algebra of $A$. If the braiding $R_{A}, A$ is the twist map this reduces to the opposite algebra as usually defined. Another basic construction is the "braided tensor product" of algebras.

Lemma 2. Let $A$ and $B$ be algebras in $\mathscr{V}$ with products $m_{A}, m_{B}$ and unit maps $l_{A}, l_{B}$, respectively. Endowed with the multiplication map

$$
M=\left(m_{A} \otimes m_{B}\right)\left(\mathrm{id}_{A} \otimes R_{B, A} \otimes \mathrm{id}_{B}\right)
$$

and the unit map $\imath=l_{A} \otimes l_{B}, A \otimes B$ is an algebra in $\mathscr{V}$. (Here we are identifying $l_{A} \otimes l_{B}: k \otimes k \rightarrow A \otimes B$ with a map from $k$ to $A \otimes B$.)

Proof. First we check associativity, as shown in Figure 6.

Step 1: Starting with

$$
\begin{array}{r}
M\left(\mathrm{id}_{A \otimes B} \otimes M\right)=\left(m_{A} \otimes m_{B}\right)\left(\mathrm{id}_{A} \otimes R_{B, A} \otimes \mathrm{id}_{B}\right) \\
\left(\operatorname{id}_{A \otimes B} \otimes m_{A} \otimes m_{B}\right)\left(\mathrm{id}_{A \otimes B \otimes A} \otimes R_{B, A} \otimes \mathrm{id}_{B}\right),
\end{array}
$$



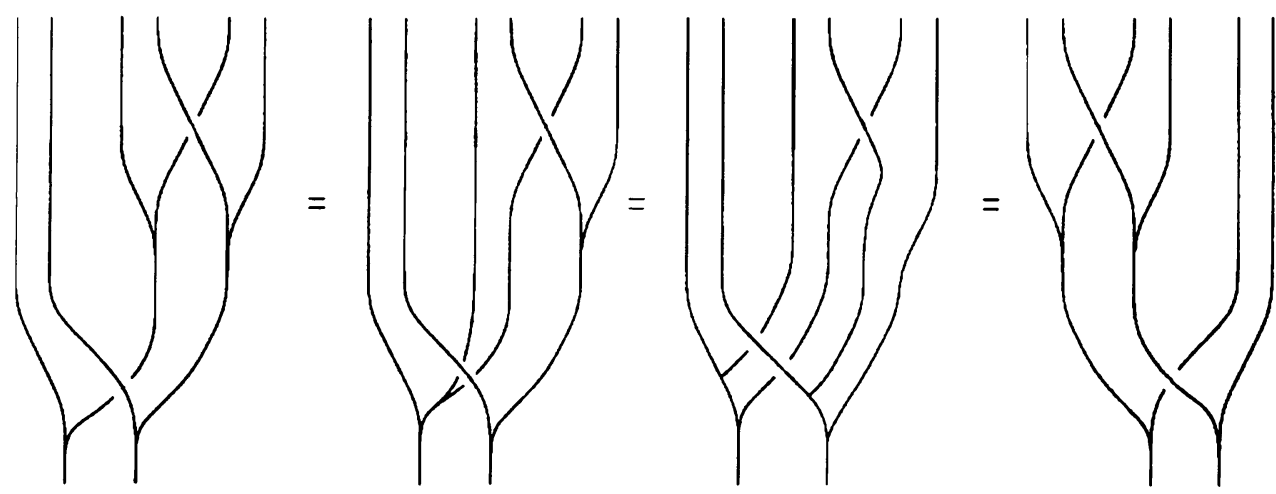

FIGURE 6. Associativity of the braided tensor product

and using quasitriangularity, we obtain

$\left(m_{A} \otimes m_{B}\right)\left(\mathrm{id}_{A} \otimes m_{A} \otimes \mathrm{id}_{B} \otimes m_{B}\right)\left(\mathrm{id}_{A^{\otimes 2}} \otimes R_{B, A} \otimes \mathrm{id}_{B^{\otimes 2}}\right)\left(\mathrm{id}_{A} \otimes R_{B, A} \otimes R_{B, A} \otimes \mathrm{id}_{B}\right)$.

Step 2: Using associativity twice, we obtain

$\left(m_{A} \otimes m_{B}\right)\left(m_{A} \otimes \mathrm{id}_{A} \otimes m_{B} \otimes \mathrm{id}_{B}\right)\left(\mathrm{id}_{A^{\otimes 2}} \otimes R_{B, A} \otimes \mathrm{id}_{B \otimes 2}\right)\left(\mathrm{id}_{A} \otimes R_{B, A} \otimes R_{B, A} \otimes \mathrm{id}_{B}\right)$.

Step 3: Using quasitriangularity again, this equals

$\left(m_{A} \otimes m_{B}\right)\left(\mathrm{id}_{A} \otimes R_{B, A} \otimes \mathrm{id}_{B}\right)\left(m_{A} \otimes m_{B} \otimes \mathrm{id}_{A \otimes B}\right)\left(\mathrm{id}_{A} \otimes R_{B, A} \otimes \mathrm{id}_{B \otimes A \otimes B}\right)$

or $M\left(M \otimes \mathrm{id}_{A \otimes B}\right)$.

To show that $l$ is a unit map for $M$ we note that

$$
M\left(l \otimes \mathrm{id}_{A \otimes B}\right)=\left(m_{A} \otimes m_{B}\right)\left(l_{A} \otimes\left(\mathrm{id}_{A} \otimes l_{B}\right) R_{k, A} \otimes \mathrm{id}_{B}\right)=L_{A \otimes B},
$$

and similarly $M\left(\mathrm{id}_{A \otimes B} \otimes l\right)=r_{A \otimes B}$.

Given two algebras $A, B \in \mathscr{V}$, we call the algebra $A \otimes B$ constructed in Lemma 2 the braided tensor product of $A$ and $B$. We write this algebra as $A \widehat{\otimes} B$ to emphasize that it need not be isomorphic to the usual tensor product of the algebras $A$ and $B$. The braided tensor product generalizes the well-known $\mathbf{Z}_{2}$-graded tensor product of $\mathbf{Z}_{2}$-graded algebras. Braided tensor products are already visible in a number of other contexts as well. For example, noncommutative tori are braided tensor products of algebras of Laurent polynomials. (Geometrically speaking, one could say that a noncommutative torus is a braided product of circles.) Also, the algebra of differential forms on a quantum vector space with Hecke-type relations is the braided tensor product of an " $r$-symmetric" and an " $r$-exterior" algebra [2].

One surprise is that the braided tensor product of $r$-commutative algebras in $\mathscr{V}$ need not be $r$-commutative, although this is always true when $\mathscr{V}$ is symmetric. More precisely:

Lemma 3. Let $A$ and $B$ be r-commutative algebras in $\mathscr{V}$ and suppose $R_{B, A} R_{A, B}=\mathrm{id}_{A \otimes B}$. Then $A \widehat{\otimes} B$ is r-commutative.

Proof. The proof is shown in Figure 7. Let $M$ denote the multiplication map for $A \widehat{\otimes} B$. Then we need to show that $M=M R_{A \otimes B}, A \otimes B$. By repeated use of the hexagon axioms we have

$$
R_{A \otimes B, A \otimes B}=\left(\mathrm{id}_{A} \otimes R_{A, B} \otimes \mathrm{id}_{B}\right)\left(R_{A, A} \otimes R_{B, B}\right)\left(\mathrm{id}_{A} \otimes R_{B, A} \otimes \mathrm{id}_{B}\right) .
$$



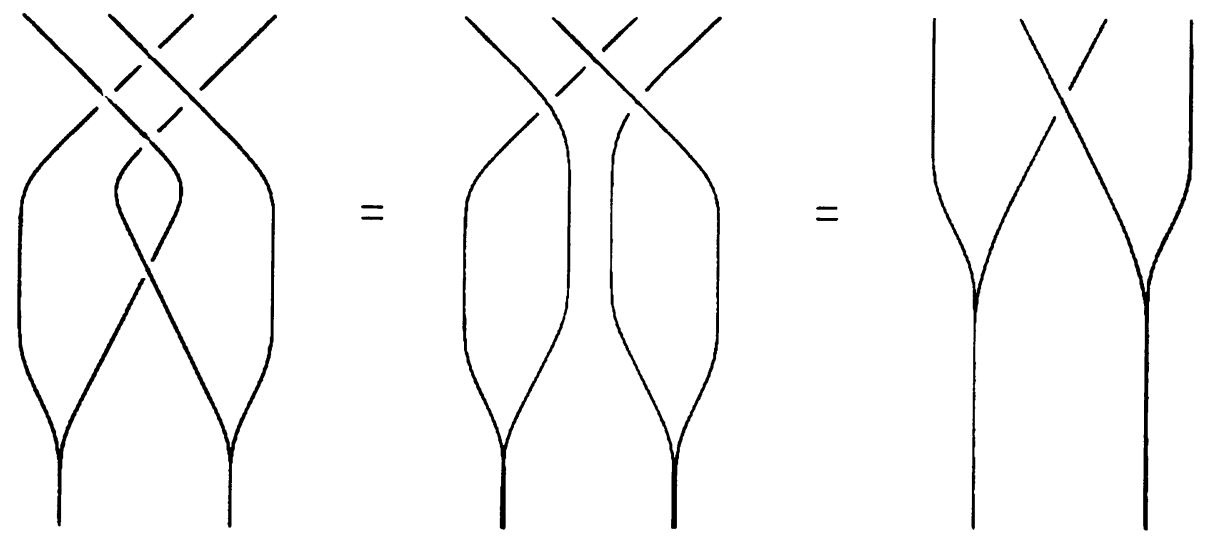

FIgURE 7. $R$-commutativity of the braided tensor product of $r$-commutative algebras

Using the fact that $R_{B, A} R_{A, B}=\mathrm{id}_{A \otimes B}$, it follows that

$$
M R_{A \otimes B, A \otimes B}=\left(m_{A} \otimes m_{B}\right)\left(R_{A, A} \otimes R_{B, B}\right)\left(\mathrm{id}_{A} \otimes R_{B, A} \otimes \mathrm{id}_{B}\right) .
$$

Using the $r$-commutativity of $A$ and $B$, it follows that

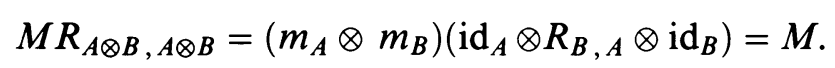

Given an algebra $A$ in $\mathscr{V}$, we define a left A-module in $\mathscr{V}$ to be an object $E$ of $\mathscr{V}$ together with a left action, a morphism $\alpha \in \operatorname{Hom}_{\mathscr{V}}(A \otimes E, E)$ satisfying

$$
\alpha\left(\mathrm{id}_{A} \otimes \alpha\right)=\alpha\left(m \otimes \mathrm{id}_{E}\right) \quad \text { and } \quad \alpha\left(l \otimes \mathrm{id}_{E}\right)=l_{E} .
$$

Given two left $A$-modules $(E, \alpha)$ and $(F, \beta)$ in $\mathscr{V}$, we define a left $A$ module morphism in $\mathscr{V}$ to be a morphism $f \in \operatorname{Hom}_{\mathscr{V}}(E, F)$ such that $f \alpha=$ $\beta\left(\operatorname{id}_{A} \otimes f\right)$. Right $A$-modules and right $A$-module morphisms in $\mathscr{V}$ are defined analogously.

Let $A^{e}$ denote the braided tensor product $A \widehat{\otimes} A^{\mathrm{op}}$.

Lemma 4. Let $A$ be an algebra in $\mathscr{V}$. Then $A$ is a left $A^{e}$-module in $\mathscr{V}$ with the left action $\lambda \in \operatorname{Hom}_{\mathscr{V}}\left(A^{e} \otimes A, A\right)$ given by $\lambda=m\left(\mathrm{id}_{A} \otimes m R_{A, A}\right)$.

Proof. Let $R$ denote $R_{A, A}$ and let $M$ denote the multiplication map of $A^{e}$ :

$$
M=(m \otimes m R)\left(\mathrm{id}_{A} \otimes R \otimes \mathrm{id}_{A}\right) .
$$

We need to show that $\lambda\left(\operatorname{id}_{A^{e}} \otimes \lambda\right)=\lambda\left(M \otimes \mathrm{id}_{A}\right)$. The proof of this is shown in Figure 8 . We begin by considering

$$
\begin{aligned}
\lambda\left(\mathrm{id}_{A^{e}} \otimes \lambda\right) & =m\left(\mathrm{id}_{A} \otimes m R\right)\left(\mathrm{id}_{A^{e}} \otimes m\left(\mathrm{id}_{A} \otimes m R\right)\right) \\
& =m\left(\mathrm{id}_{A} \otimes m\right)\left(\mathrm{id}_{A} \otimes R\left(\mathrm{id}_{A} \otimes m\right)\right)\left(\mathrm{id}_{A^{\otimes 3}} \otimes m R\right) .
\end{aligned}
$$

Step 1: By quasitriangularity this equals

$$
\begin{aligned}
& m\left(\mathrm{id}_{A} \otimes m\right)\left(\mathrm{id}_{A} \otimes\left(m \otimes \mathrm{id}_{A}\right)\left(\mathrm{id}_{A} \otimes R\right)\left(R \otimes \mathrm{id}_{A}\right)\right)\left(\mathrm{id}_{A^{\otimes 3}} \otimes m R\right) \\
& \quad=m\left(\mathrm{id}_{A} \otimes m\right)\left(\mathrm{id}_{A} \otimes m \otimes \mathrm{id}_{A}\right)\left(\mathrm{id}_{A^{\otimes 2}} \otimes R\left(\mathrm{id}_{A} \otimes m\right)\right)\left(\mathrm{id}_{A} \otimes R \otimes R\right) .
\end{aligned}
$$

Step 2: By quasitriangularity this equals

$m\left(\mathrm{id}_{A} \otimes m\right)\left(\mathrm{id}_{A} \otimes m \otimes \mathrm{id}_{A}\right)\left(\mathrm{id}_{A^{\otimes 2}} \otimes\left(m \otimes \mathrm{id}_{A}\right)\left(\mathrm{id}_{A} \otimes R\right)\left(R \otimes \mathrm{id}_{A}\right)\right)\left(\mathrm{id}_{A} \otimes R \otimes R\right)$. 

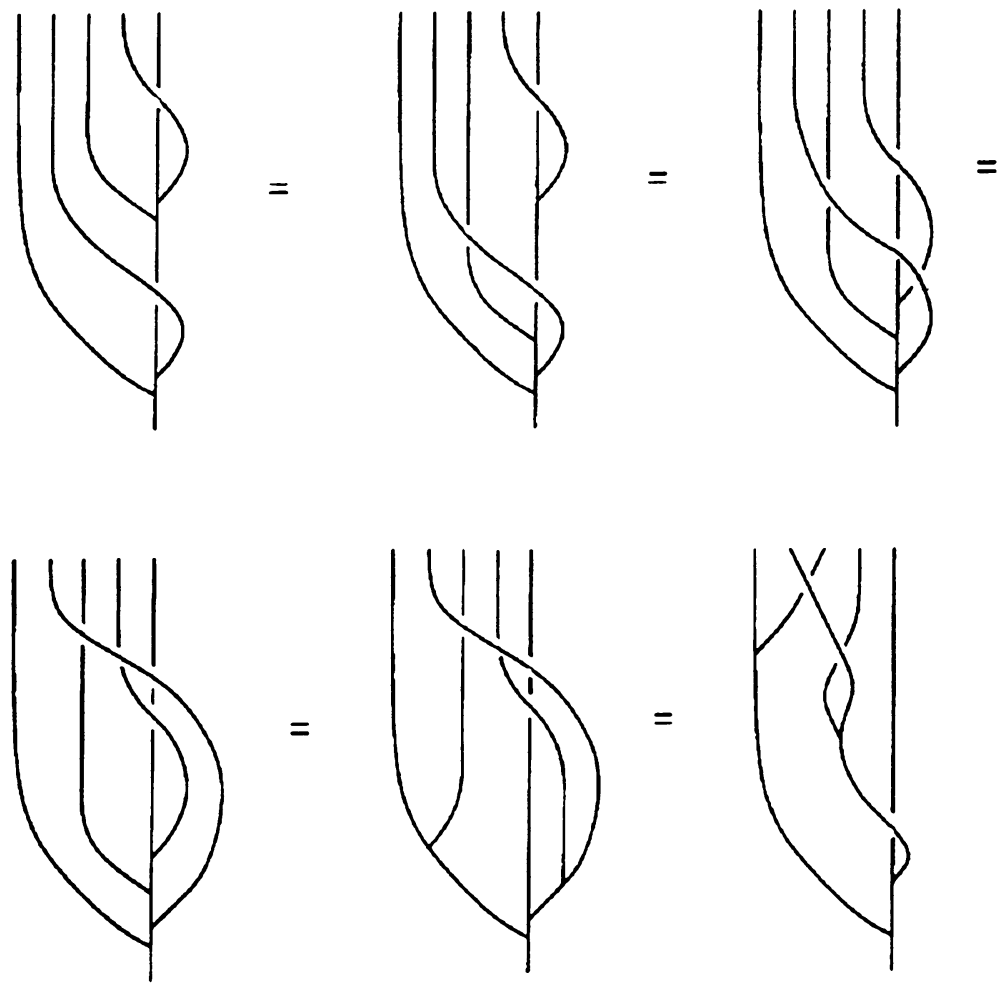

Figure 8. $A$ is a left $A^{e}$-module

Step 3: By the Yang-Baxter equation this equals

$$
\begin{aligned}
& m\left(\mathrm{id}_{A} \otimes m\right)\left(\mathrm{id}_{A} \otimes m \otimes \mathrm{id}_{A}\right)\left(\mathrm{id}_{A \otimes 2} \otimes m R \otimes \mathrm{id}_{A}\right) \\
& \quad\left(\mathrm{id}_{A^{\otimes 3}} \otimes R\right)\left(\mathrm{id}_{A^{\otimes 2}} \otimes R \otimes \mathrm{id}_{A}\right)\left(\mathrm{id}_{A} \otimes R \otimes \mathrm{id}_{A^{\otimes 2}}\right) .
\end{aligned}
$$

Step 4: Using associativity three times, this equals

$$
\begin{aligned}
& m\left(\mathrm{id}_{A} \otimes m\right)\left(m \otimes \mathrm{id}_{A} \otimes m\right)\left(\mathrm{id}_{A^{\otimes 2}} \otimes R \otimes \mathrm{id}_{A}\right)\left(\mathrm{id}_{A^{\otimes 3}} \otimes R\right) \\
& \quad\left(\mathrm{id}_{A^{\otimes 2}} \otimes R \otimes \mathrm{id}_{A}\right)\left(\mathrm{id}_{A} \otimes R \otimes \mathrm{id}_{A^{\otimes 2}}\right) .
\end{aligned}
$$

Step 5: By quasitriangularity this equals

$$
\begin{gathered}
m\left(\mathrm{id}_{A} \otimes m\right)(m \otimes R)\left(\mathrm{id}_{A^{\otimes 2}} \otimes m \otimes \mathrm{id}_{A}\right)\left(\mathrm{id}_{A^{\otimes 2}} \otimes R \otimes \mathrm{id}_{A}\right)\left(\mathrm{id}_{A} \otimes R \otimes \mathrm{id}_{A^{\otimes 2}}\right) \\
\quad=m\left(\mathrm{id}_{A} \otimes m R\right)\left(m \otimes m R \otimes \mathrm{id}_{A}\right)\left(\mathrm{id}_{A} \otimes R \otimes \mathrm{id}_{A^{\otimes 2}}\right) .
\end{gathered}
$$

The latter expression equals $\lambda\left(M \otimes \mathrm{id}_{A}\right)$, as was to be shown.

To complete the proof that $\lambda$ is a left action it suffices to note that $l \otimes l: k \rightarrow$ $A \otimes A$ is the unit map for $A^{e}$, and

$$
\begin{aligned}
\lambda\left(l \otimes l \otimes \mathrm{id}_{A}\right) & =m\left(\mathrm{id}_{A} \otimes m R\right)\left(l \otimes l \otimes \mathrm{id}_{A}\right) \\
& =m\left(l \otimes m\left(\mathrm{id}_{A} \otimes l\right) R_{k, A}\right) \\
& =m\left(l \otimes r_{A} R_{k, A}\right)=m\left(l \otimes l_{A}\right)=l_{A} .
\end{aligned}
$$

Unfortunately, there is no right action of $A^{e}$ on $A$ analogous to the left action unless we introduce a further condition. Let us say that an $r$-algebra $A$ in $\mathscr{V}$ is weakly r-commutative if the multiplication map $m: A \otimes A \rightarrow A$ satisfies $m=m R_{A, A}^{2}$. Note that this is the case if $A$ is either $r$-commutative or strong, i.e., $R_{A, A}^{2}=\mathrm{id}_{A \otimes A}$. 
Lemma 5. Let $A$ be a weakly $r$-commutative algebra in $\mathscr{V}$. Then $A$ is a right $A^{e}$-module in $\mathscr{V}$ with the right action $\rho \in \operatorname{Hom}_{\mathscr{V}}\left(A \otimes A^{e}, A\right)$ given by

$$
\rho=m R_{A, A}\left(m \otimes \mathrm{id}_{A}\right) .
$$

Proof. Writing $R$ for $R_{A, A}$ and $M$ for the multiplication map of $A^{e}$, we need to show that $\rho\left(\rho \otimes \mathrm{id}_{A^{e}}\right)=\rho\left(\mathrm{id}_{A} \otimes m\right)$. We begin by using weak $r$ commutativity to write

$$
\rho\left(\rho \otimes \mathrm{id}_{A^{e}}\right)=m R^{-1}\left(m \otimes \mathrm{id}_{A}\right)\left(m R^{-1}\left(m \otimes \mathrm{id}_{A}\right) \otimes \mathrm{id}_{A^{\otimes 2}}\right) .
$$

By a sequence of six steps shown in Figure 9, this equals

$$
m R^{-1}\left(\mathrm{id}_{A} \otimes m \otimes m R^{-1}\right)\left(\mathrm{id}_{A^{\otimes 2}} \otimes R \otimes \mathrm{id}_{A}\right) .
$$

These steps use: (1) associativity, (2) quasitriangularity, (3) quasitriangularity, (4) associativity (twice), (5) the Yang-Baxter equation, and (6) quasitriangularity. By weak $r$-commutativity the result is equal to

$$
m R\left(\mathrm{id}_{A} \otimes m \otimes m R\right)\left(\mathrm{id}_{A^{\otimes 2}} \otimes R \otimes \mathrm{id}_{A}\right),
$$

or $m\left(\mathrm{id}_{A} \otimes m\right)$.

The proof that the unit map $l \otimes l: k \rightarrow A^{e}$ satisfies $\rho\left(\mathrm{id}_{A} \otimes l \otimes l\right)=r_{A}$ is similar to the calculation at the end of the proof of Lemma 4 .
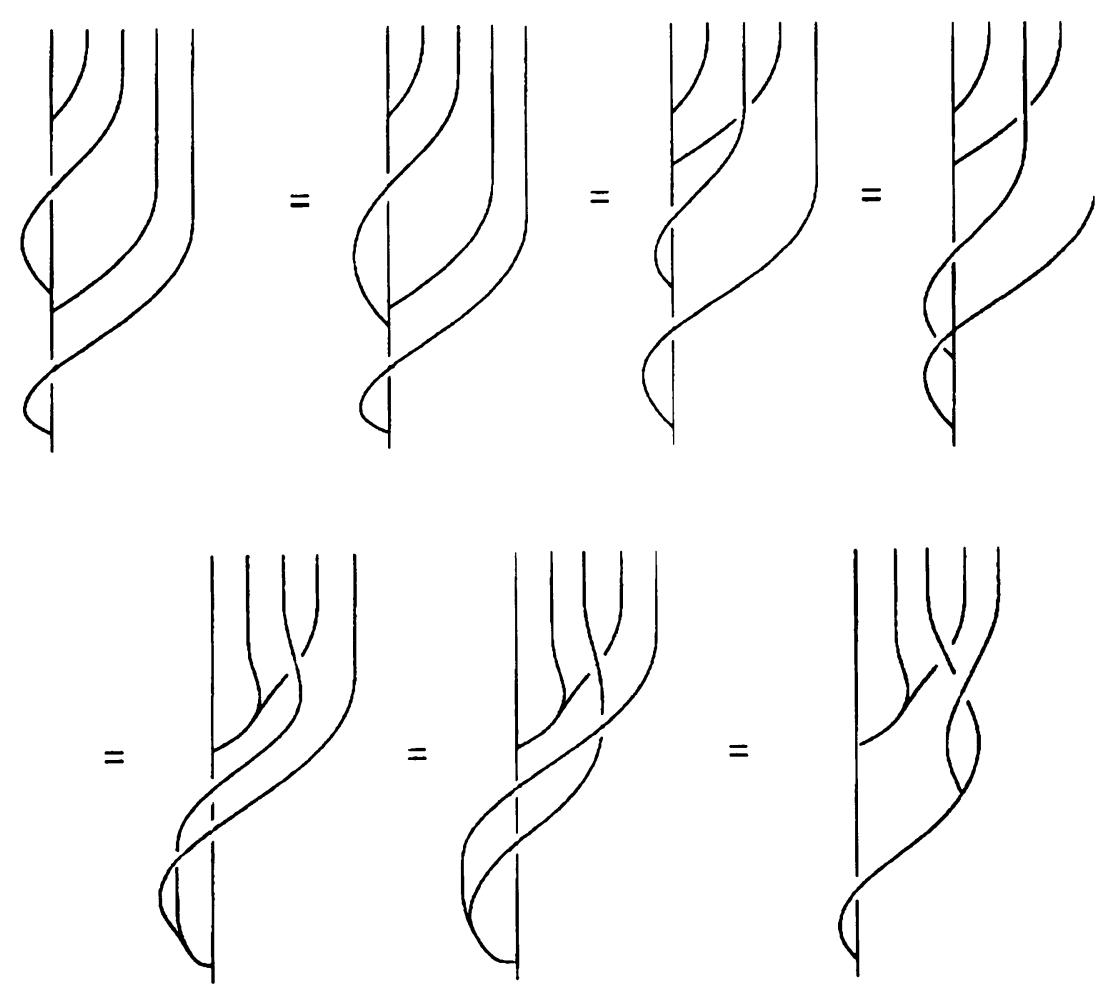

Figure 9. $A$ is a right $A^{e}$-module if $A$ is weakly $r$ commutative 


\section{BRAIDED HochSCHILD HOMOLOGY}

In this section we generalize the usual construction of Hochschild homology to an arbitrary braided tensor category. Let $k$ be a commutative ring, let $\mathscr{V}$ be a fixed braided tensor category of $k$-modules, and let $A$ be an algebra in $\mathscr{V}$. We begin by constructing a flat resolution of $A$ as a left $A^{e}$-module, under the assumption that $A$ is flat as a $k$-module.

For $n \geq 1$, let $\alpha \in \operatorname{Hom}_{\mathscr{V}}\left(A^{e} \otimes A^{\otimes n}, A^{\otimes n}\right)$ be given by $(a \otimes b) \otimes\left(a_{1} \otimes \cdots \otimes a_{n}\right) \mapsto\left(m \otimes \mathrm{id}_{A^{\otimes(n-2)}} \otimes m\right)\left(\mathrm{id}_{A} \otimes R_{A, A^{\otimes n}}\right)\left(a \otimes b \otimes a_{1} \otimes \cdots \otimes a_{n}\right)$. Theorem 1. Suppose $\mathscr{V}$ a braided tensor category of $k$-modules, and $A$ an algebra in $A$. The map $\alpha$ is a left action of $A^{e}$ on $A^{\otimes n}$. If $A$ is flat over $k$, $A^{\otimes n}$ is a flat left $A^{e}$-module for $n \geq 2$. Defining $d_{n} \in \operatorname{Hom}_{\mathscr{V}}\left(A^{\otimes n}, A^{\otimes(n-1)}\right)$ for $n \geq 2$ by

$$
d_{n}\left(a_{1} \otimes \cdots \otimes a_{n}\right)=\sum_{i=1}^{n-1}(-1)^{i+1} a_{1} \otimes \cdots \otimes a_{i} a_{i+1} \otimes \cdots \otimes a_{n},
$$

then

$$
\cdots \rightarrow A^{\otimes 3} \stackrel{d_{3}}{\rightarrow} A^{\otimes 2} \stackrel{d_{2}}{\rightarrow} A \rightarrow 0
$$

is a long exact sequence of left $A^{e}$-module morphisms in $\mathscr{V}$.

Proof. First, define $K_{n}$ for $n \geq-1$ by

$$
K_{n}= \begin{cases}A^{e} \otimes A^{\otimes n}, & n \geq 1, \\ A^{e}, & n=0, \\ A, & n=-1 .\end{cases}
$$

We give $K_{n}$ the structure of a left $A^{e}$-module in $\mathscr{V}$ as follows. Define $\beta \in$ $\operatorname{Hom}_{\mathscr{V}}\left(A^{e} \otimes K_{n}, K_{n}\right)$ by

$$
\beta= \begin{cases}M \otimes \mathrm{id}_{A^{\otimes n}}, & n \geq 1, \\ M, & n=0, \\ \lambda, & n=-1,\end{cases}
$$

where $M$ is a multiplication map for $A^{e}$, and $\lambda$ is the left action of $A^{e}$ on $A$. Then $\beta$ is a left action of $A^{e}$ on $K_{n}$ by Lemmas 1,2 , and 4 . Note that if $A$ is a flat $k$-module, $K_{n}$ is a flat $A^{e}$-module for $n \geq 0$. Next, define $T \in \operatorname{Hom}_{\mathscr{V}}\left(K_{n}, A^{\otimes(n+2)}\right)$ for $n \geq-1$ by

$$
T= \begin{cases}\mathrm{id}_{A} \otimes R_{A}, A^{\otimes n}, & n \geq 1, \\ \mathrm{id}_{A^{e}}, & n=0, \\ \mathrm{id}_{A}, & n=-1 .\end{cases}
$$

A calculation shows that $\alpha=T \beta\left(\mathrm{id}_{A^{e}} \otimes T^{-1}\right)$. It follows that $\alpha$ is a left action of $A^{e}$ on $A^{\otimes n}$, and that if $A$ is flat then $A^{\otimes n}$ is a flat left $A^{e}$-module for $n \geq 2$.

Exactness of the sequence

$$
\cdots \rightarrow A^{\otimes 3} \stackrel{d_{3}}{\rightarrow} A^{\otimes 2} \stackrel{d_{2}}{\rightarrow} A \rightarrow 0
$$

is well-known [3], so the only thing left to show is that the maps $d_{n}$ are morphisms of $A^{e}$-modules. It suffices to show that each map

$$
a_{1} \otimes \cdots \otimes a_{n} \mapsto a_{1} \otimes \cdots \otimes a_{i} a_{i+1} \otimes \cdots \otimes a_{n}
$$


for $i=1, \ldots, n-1$, is a morphism of $A^{e}$-modules in $\mathscr{V}$. For $1<i<n-1$, this follows directly from quasitriangularity, while for $i=1$ and $i=n-1$ one must also use associativity.

Given an algebra $A$ in $\mathscr{V}$ we define the acyclic braided Hochschild complex $K(A)$ to be the chain complex of left $A^{e}$-modules $\left(K_{n}(A), b^{\prime}\right)(n \geq 0)$, where

$$
K_{n}(A)=A^{e} \otimes A^{\otimes n},
$$

taking $A^{\otimes 0}=k$, and

$$
b_{n}^{\prime}=T d_{n+2} T^{-1},
$$

with $T: A^{\otimes(n+2)} \rightarrow K_{n}$ given by equation (1). Note that this complex really depends only on the structure of $A$ as an $r$-algebra, not on the braided tensor category in which $A$ is an algebra.

Corollary 1. The chain complex of left $A^{e}$-modules $\left(A^{\otimes(n+2)}, d_{n+2}\right)$ (where $n \geq$ $0)$ is isomorphic to $K(A)$ by $T: A^{\otimes(n+2)} \rightarrow K_{n}(A)$.

Proof. This follows from the proof of Theorem 1.

Corollary 2. Let $A$ be an algebra in $\mathscr{V}$, and let $E$ be a right $A^{e}$-module in $\mathscr{V}$. Then there is a natural isomorphism between $\operatorname{Tor}_{n}^{A^{e}}(E, A)$ and $H_{n}\left(E \otimes_{A^{e}} K(A)\right)$. Proof. This follows from Theorem 1 using homological algebra [3].

Now suppose that $A$ is weakly $r$-commutative. Then $A$ has the structure of a right $A^{e}$-module in $\mathscr{V}$ by Lemma 5 . Note that in this case the chain complex of Corollary 1 computing $\operatorname{Tor}_{n}^{A^{e}}(A, A)$ depends only on the structure of $A$ as an $r$-algebra. We call this complex the braided Hochschild complex of $A, C(A)=\left(C_{n}(A), b_{n}\right)$, where

$$
C_{n}(A)=A \otimes_{A^{e}} K_{n}(A)
$$

and $b_{n}=\mathrm{id}_{A} \otimes b_{n}^{\prime}$. We call the homology of this complex the braided Hochschild homology of $A$, which we denote as $H^{R}(A)$.

Next we define a shuffle product on the braided Hochschild complex when $A$ is a strong $r$-commutative algebra. Together with the differential of $C(A)$, this will make $C(A)$ into a differential graded algebra (with differential of degree $-1)$. Let $s_{i}, 1 \leq i<n$, denote the standard generators of the braid group $B_{n}$, which satisfy the relations

$$
s_{i} s_{j}=s_{j} s_{i}, \quad|i-j| \geq 2, \quad s_{i} s_{i+1} s_{i}=s_{i+1} s_{i} s_{i+1} .
$$

Let $\pi: B_{n} \rightarrow S_{n}$ denote the canonical surjection onto the symmetric group, which maps $s_{n}$ to the elementary transposition $\sigma_{n}$. Given $\beta \in B_{n}$, we write $\operatorname{simply} \operatorname{sign}(\beta)$ for $\operatorname{sign}(\pi(\beta))$. The notion of "shuffles" generalizes from the symmetric group to the braid group as follows. Suppose that $p+q=n$. Beginning with $p$ red strands to the left of $q$ blue strands, a braid in which at every crossing a red strand crosses over and to the right of a blue strand is called a $(p, q)$-shuffle. More algebraically, we define $\beta \in B_{n}$ to be a $(p, q)$-shuffle if it is of the form

$$
\left(s_{1+i_{1}} \cdots s_{2} s_{1}\right)\left(s_{2+i_{2}} \cdots s_{3} s_{2}\right) \cdots\left(s_{p+i_{p}} \cdots s_{p+1} s_{p}\right)
$$

where $-1 \leq i_{j} \leq n-j-1, i_{j} \leq i_{j+1}$, and for $i_{j}=-1$ we interpret the (empty) product $s_{j+i_{j}} \cdots s_{j+1} s_{j}$ as $1 \in B_{n}$. We denote the set of $(p, q)$-shuffles 
by $\operatorname{sh}(p, q)$. The canonical map from $B_{n}$ to the symmetric group $S_{n}$ defines a one-to-one correspondence between $\operatorname{sh}(p, q)$ and the elements of $S_{n}$ that are normally referred to as $(p, q)$-shuffles.

Now let $V$ be an object in $\mathscr{V}$. There is a unique representation of $B_{n}$ on $V^{\otimes n}$ such that

$$
s_{i}\left(v_{1} \otimes \cdots \otimes v_{n}\right)=v_{1} \otimes \cdots \otimes R\left(v_{i} \otimes v_{i+1}\right) \otimes \cdots \otimes v_{n} .
$$

Let $T V=\bigoplus_{n \geq 0} V^{\otimes n}$. We define the shuffle product $[\cdot ; \cdot]$ on $T V$ as follows:

Lemma 6. If $V$ is an object in $\mathscr{V}$, there is a graded associative product on $T V$ given by

$\left[v_{1} \otimes \cdots \otimes v_{p} ; w_{1} \otimes \cdots \otimes w_{q}\right]=\sum_{\beta \in \operatorname{sh}(p, q)} \operatorname{sign}(\beta) \beta\left(v_{1} \otimes \cdots \otimes v_{p} \otimes w_{1} \otimes \cdots \otimes w_{q}\right)$.

Proof. First note that given $p, q, r$ with $p+q+r=n$, there are inclusions of $B_{p}, B_{q}$, and $B_{r}$ in $B_{n}$, given by $s_{i} \mapsto s_{i}, s_{i} \mapsto s_{i+p}$, and $s_{i} \mapsto s_{i+p+q}$, respectively. Similarly, there are inclusions of $B_{p+q}$ and $B_{q+r}$ in $B_{n}$ given by $s_{i} \mapsto s_{i}$ and $s_{i} \mapsto s_{i+p}$. Identifying by these inclusions, one can show using the braid group relations that

$$
\operatorname{sh}(p+q, r) \operatorname{sh}(p, q)=\operatorname{sh}(p, q+r) \operatorname{sh}(q, r) .
$$

Associativity of product given above then follows as for the standard shuffle product.

Lemma 7. Suppose that $V$ is an object in $\mathscr{V}$ with $R_{V, V}^{2}=\mathrm{id}_{V \otimes V}$. Let $m: T V \otimes T V \rightarrow T V$ be given by $m(x \otimes y)=[x ; y]$. Then if $x \in V^{\otimes p}$ and $y \in V^{\otimes q}$,

$$
m(x \otimes y)=(-1)^{p q} m R_{V \otimes p, V \otimes q}(x \otimes y) .
$$

Proof. Since $R_{V, V}^{2}$ is the identity, the representation of $B_{n}$ on $V^{\otimes n}$ factors through a representation of $S_{n}$ and the proof follows the usual proof of graded commutativity of the shuffle product [28].

Identifying $K(A)$ with $A^{e} \widehat{\otimes} T A$, where $T A$ is given the shuffle product, $K(A)$ inherits the structure of an algebra in $\mathscr{V}$. Note that $K(A)$ is a graded algebra.

Theorem 2. Let $A$ be a strong $r$-commutative algebra over a commutative ring $k$, let $K=K(A)$ be the acyclic braided Hochschild complex of $A$, and let $m$ denote the multiplication map for $K$. Then $K$ is a differential graded algebra with differential $b^{\prime}$ of degree -1 , and for all $x \in K_{p}$ and $y \in K_{q}$,

$$
m(x \otimes y)=(-1)^{p q} m R_{K_{p}, K_{q}}(x \otimes y) .
$$

Proof. The algebra $A^{e}$ is $r$-commutative by Lemma 3. Equation (2) then follows from Lemma 7 and the method of proof of Lemma 3.

We will show that for all $x \in K_{p}$ and $y \in K_{q}$,

$$
b^{\prime}(x y)=b^{\prime}(x) y+(-1)^{p} x b^{\prime}(y) .
$$

Note that if this is known for given values of $p, q$, then

$$
b^{\prime} m R_{K_{q}, K_{p}}(x \otimes y)=m\left(b^{\prime} \otimes \mathrm{id}+(-1)^{p} \mathrm{id} \otimes b^{\prime}\right) R_{K_{q}, K_{p}}(x \otimes y)
$$


for all $x \in K_{q}$ and $y \in K_{p}$. By equation (2) and quasitriangularity, it follows that

$$
b^{\prime}(x y)=b^{\prime}(x) y+(-1)^{q} x b^{\prime}(y)
$$

Thus in what follows we assume $p \leq q$.

By Theorem 1 and Corollary $1, b^{\prime}$ is $A^{e}$-linear. Using this together with quasitriangularity, it follows that equation (3) for given $x, y$ implies

$$
b^{\prime}((e x)(f y))=b^{\prime}(e x)(f y)+(-1)^{p}(e x) b^{\prime}(f y)
$$

for all $e, f \in A^{e}$. Thus it suffices to prove equation (3) for $x, y \in K$ of the form

$$
\begin{gathered}
x=(1 \otimes 1) \otimes a_{1} \otimes \cdots \otimes a_{p}, \\
y=(1 \otimes 1) \otimes a_{p+1} \otimes \cdots \otimes a_{p+q} .
\end{gathered}
$$

We prove this by induction on $n=p+q$, following Seibt's argument in the standard case [28].

The cases $n=0,1$ are trivial. For $n=2$, the only nontrivial case occurs for $p=q=1$, where

$$
x y=1 \otimes 1 \otimes\left(a_{1} \otimes a_{2}-R\left(a_{1} \otimes a_{2}\right)\right),
$$

hence by $r$-commutativity

$$
b^{\prime}(x y)=a_{1} \otimes 1 \otimes a_{2}+1 \otimes R_{A, A}^{-1}\left(a_{1} \otimes a_{2}\right)-R_{A^{\otimes 2, A} A}\left(1 \otimes a_{1} \otimes a_{2}\right)-1 \otimes a_{1} \otimes a_{2} .
$$

On the other hand,

$$
b^{\prime}(x) y=a_{1} \otimes 1 \otimes a_{2}-1 \otimes a_{1} \otimes a_{2}
$$

and

$$
-x b^{\prime}(y)=-R_{A^{\otimes 2}, A}\left(1 \otimes a_{1} \otimes a_{2}\right)+1 \otimes R_{A, A}\left(a_{1} \otimes a_{2}\right),
$$

so using the fact that $A$ is strong, equation (3) holds in this case.

Now assuming the inductive hypothesis for $n-1 \geq 2$, we will prove it for $n$. First, though, let $s: K_{k} \rightarrow K_{k+1}$ be given by

$$
s\left(a_{-1} \otimes a_{0} \otimes \cdots \otimes a_{k}\right)=\left(1 \otimes R_{A, A}^{-1}\left(a_{-1} \otimes a_{0}\right) \otimes a_{1} \otimes \cdots \otimes a_{k}\right) .
$$

An easy calculation (not using the fact that $A$ is strong or $r$-commutative) shows that $s$ is a contracting homotopy for $K$, i.e.,

$$
s b^{\prime}+b^{\prime} s=\mathrm{id}_{K} .
$$

Next, note that the left $A^{e}$-module structure of $K$ and the natural homomorphism $A \rightarrow A^{e}$ make $K$ into a left $A$-module, and that the product in $K$ satisfies $a(u v)=(a u) v$ for all $a \in A$ and $u, v \in K$.

Now let

$$
\begin{gathered}
\bar{x}=(1 \otimes 1) \otimes a_{2} \otimes \cdots \otimes a_{p} \in K_{p-1}, \\
\bar{y}=(1 \otimes 1) \otimes a_{p+2} \otimes \cdots \otimes a_{n} \in K_{q-1} .
\end{gathered}
$$

We prove the induction hypothesis, equation (3), using the following identities, which we verify below:

$$
\begin{gathered}
x y=s\left(a_{1} \bar{x} y+(-1)^{p} x\left(a_{p+1} \bar{y}\right)\right) \\
b^{\prime}(x) y=a_{1} \bar{x} y-s\left(a_{1} b^{\prime}(\bar{x}) y+(-1)^{p} b^{\prime}(x) a_{p+1} \bar{y}\right), \\
x b^{\prime}(y)=x a_{p+1} \bar{y}+s\left(a_{1} \bar{x} b^{\prime}(y)-(-1)^{p} x a_{p+1} b^{\prime}(\bar{y})\right) .
\end{gathered}
$$


Using equations (4) and (5),

$$
b^{\prime}(x y)=a_{1} \bar{x} y+(-1)^{p} a_{p+1} x \bar{y}-s b^{\prime}\left(a_{1} \bar{x} y+(-1)^{p} x a_{p+1} \bar{y}\right) .
$$

Using the fact that $b^{\prime}$ is a left $A$-module morphism, together with the induction hypothesis, we have

$$
\begin{aligned}
b^{\prime}(x y)= & a_{1} \bar{x} y+(-1)^{p} x a_{p+1} \bar{y}-s\left(a_{1} b^{\prime}(\bar{x}) y\right)+(-1)^{p} s\left(a_{1} \bar{x} b^{\prime}(y)\right) \\
& -(-1)^{p} s\left(b^{\prime}(x) a_{p+1} \bar{y}\right)-s\left(x a_{p+1} b^{\prime}(\bar{y})\right) .
\end{aligned}
$$

Equation (3) now follows from equations (6) and (7).

To prove equation (5), note that

$$
s\left(a_{1} \bar{x} y\right)=(1 \otimes 1) \otimes \sum_{\beta \in \operatorname{sh}(p-1, q)} \operatorname{sign}(\beta) a_{1} \otimes \beta\left(a_{2} \otimes \cdots \otimes a_{n}\right)
$$

and

$$
s\left(x a_{p+1} \bar{y}\right)=(1 \otimes 1) \otimes \sum_{\beta \in \operatorname{sh}(p, q-1)} \operatorname{sign}(\beta)\left(\operatorname{id}_{A} \otimes \beta\right)\left(R_{A^{\otimes p}, A} \otimes \mathrm{id}_{A^{\otimes(q-1)}}\right)\left(a_{1} \otimes \cdots \otimes a_{n}\right) .
$$

Let $i: B_{n-1} \rightarrow B_{n}$ denote the inclusion given by

$$
i\left(s_{k}\right)=s_{k+1} \text {. }
$$

Define the one-to-one map $j: B_{n-1} \rightarrow B_{n}$ (not a homomorphism) by

$$
j(\beta)=\beta s_{1} \cdots s_{p} \text {. }
$$

We have

$$
\begin{aligned}
& s\left(a_{1} \bar{x} y\right)=(1 \otimes 1) \otimes \sum_{\beta \in \operatorname{sh}(p-1, q)} \operatorname{sign}(\beta) i(\beta)\left(a_{1} \otimes \cdots \otimes a_{n}\right), \\
& s\left(x a_{p+1} \bar{y}\right)=(1 \otimes 1) \otimes \sum_{\beta \in \operatorname{sh}(p, q-1)} \operatorname{sign}(\beta) j(\beta)\left(a_{1} \otimes \cdots \otimes a_{n}\right) .
\end{aligned}
$$

Note that $i(\operatorname{sh}(p-1, q))$ and $j(\operatorname{sh}(p, q-1))$ are contained in $\operatorname{sh}(p, q)$. Moreover, $i(\operatorname{sh}(p-1, q))$ and $j(\operatorname{sh}(p, q-1))$ are disjoint, since if $s=\pi(\beta) \in S_{n}$ is the permutation corresponding to $\beta \in i(\operatorname{sh}(p-1, q))$ then $s(1)=1$, while if $s=\pi(\beta)$ for $\beta \in j(\operatorname{sh}(p, q-1))$ then $s(p+1)=1$. Since $\operatorname{sh}(p, q)$ has $(p+q) ! / p ! q$ ! elements, a counting argument shows that $\operatorname{sh}(p, q)$ is the disjoint union of $i(\operatorname{sh}(p-1, q))$ and $j(\operatorname{sh}(p, q-1)$. Using the fact that

$$
\operatorname{sign}(i(\beta))=\operatorname{sign}(\beta), \quad \operatorname{sign}(j(\beta))=(-1)^{p} \operatorname{sign}(\beta),
$$

it follows that

$$
s\left(a_{1} \bar{x} y+(-1)^{p} x a_{p+1} \bar{y}\right)=(1 \otimes 1) \otimes \sum_{\beta \in \operatorname{sh}(p, q)} \operatorname{sign}(\beta) \beta\left(a_{1} \otimes \cdots \otimes a_{n}\right)=x y
$$

as desired.

To conclude, we prove (6), omitting the proof of (7) as it is analogous. Note first that

$$
\begin{aligned}
b^{\prime}(x)-a_{1} \bar{x}= & \sum_{i=1}^{p-1}(-1)^{i} \otimes 1 \otimes a_{1} \otimes \cdots \otimes a_{i} a_{i+1} \otimes \cdots \otimes a_{p} \\
& +(-1)^{p} \otimes R_{A^{\otimes(p-1)}, A}\left(a_{1} \otimes \cdots \otimes a_{p}\right) .
\end{aligned}
$$


(Here and below powers of -1 are taken as elements of $A$.) Thus

(8)

$$
\begin{array}{r}
b^{\prime}(x) y-a_{1} \bar{x} y \\
=\sum_{\beta \in \operatorname{sh}(p-1, q)} \operatorname{sign}(\beta)\left\{\sum_{i=1}^{p-1}(-1)^{i} \otimes 1 \otimes \beta\left(a_{1} \otimes \cdots \otimes a_{i} a_{i+1} \otimes \cdots \otimes a_{n}\right)+(-1)^{p}\right. \\
\left.\otimes\left(\mathrm{id}_{A} \otimes \beta\right)\left(R_{A^{\otimes(p-1)}, A} \otimes \mathrm{id}_{A \otimes q}\right)\left(a_{1} \otimes \cdots \otimes a_{n}\right)\right\} .
\end{array}
$$

Define $i: B_{n-2} \rightarrow B_{n-1}$ to be the homomorphism given by $i\left(s_{k}\right)=s_{k+1}$, and define $j: B_{n-2} \rightarrow B_{n-1}$ by $j(\beta)=i(\beta) s_{1} \cdots s_{p-1}$. Since $\operatorname{sh}(p-1, q)$ is the disjoint union of $i(\operatorname{sh}(p-2, q))$ and $j(\operatorname{sh}(p-1, q-1))$ we may write the right side of $(8)$ as the sum of two terms,

$$
\begin{aligned}
& X=\sum_{\beta \in \operatorname{sh}(p-2, q)} \operatorname{sign}(\beta)\{-1 \otimes 1 \otimes a_{1} a_{2} \otimes \beta\left(a_{3} \otimes \cdots \otimes a_{n}\right) \\
&+\sum_{i=2}^{p-1}(-1)^{i} \otimes 1 \otimes a_{1} \otimes \beta\left(a_{2} \otimes \cdots \otimes a_{i} a_{i+1} \otimes \cdots \otimes a_{n}\right) \\
&+(-1)^{p} \otimes\left(\mathrm{id}_{A^{\otimes 2}} \otimes \beta\right)\left(R_{A^{\otimes(p-1)}, A} \otimes \mathrm{id}_{A} \otimes q\right. \\
&
\end{aligned}
$$

and

$$
\begin{aligned}
& Y=\sum_{\beta \in \operatorname{sh}(p-1, q-1)} \operatorname{sign}(\beta)\left\{1 \otimes\left(\mathrm{id}_{A^{\otimes 2}} \otimes \beta\right)\left(R_{A^{\otimes p}, A} \otimes \mathrm{id}_{A^{\otimes(q-1)}}\right)\right. \\
& \left(R_{A^{\otimes(p-1)}, A} \otimes \mathrm{id}_{A^{\otimes q}}\right)\left(a_{1} \otimes \cdots \otimes a_{n}\right)+\sum_{i=1}^{p-1}(-1)^{i+p} \otimes 1 \\
& \left.\otimes\left(\mathrm{id}_{A} \otimes \beta\right)\left(R_{A^{\otimes p}, A} \otimes \mathrm{id}_{A^{\otimes(q-1)}}\right)\left(a_{2} \otimes \cdots \otimes a_{i} a_{i+1} \otimes \cdots \otimes a_{n}\right)\right\} .
\end{aligned}
$$

It now suffices to check that the first term equals $s\left(a_{1} b^{\prime}(\bar{x}) y\right)$, while the second equals $s\left(b^{\prime}(x) a_{p+1} \bar{y}\right)$.

Noting that the braided Hochschild complex $C(A)=A \otimes_{A^{e}} K(A)$ is the quotient of $A \otimes K(A)$ by an ideal, when the latter is regarded as the braided tensor product $A \widehat{\otimes} K(A)$, we obtain a product on $C(A)$.

Corollary 3. Let $A$ be a strong $r$-commutative algebra over a commutative ring $k$, let $C=C(A)$ be the braided Hochschild complex of $A$, and let $m$ denote the multiplication map for $C$. Then $C$ is a differential graded algebra with differential $b$ of degree -1 , and for all $x \in C_{p}$ and $y \in C_{q}$,

$$
m(x \otimes y)=(-1)^{p q} m R_{C_{p}, C_{q}}(x \otimes y) .
$$

Proof. This follows from Theorem 2. 


\section{HOCHSCHILD HOMOLOGY AND DIFFERENTIAL FORMS}

Let $A$ be a strong $r$-commutative $r$-algebra with $r$-structure $R$. By the results of the previous section the braided Hochschild homology $H^{R}(A)$ is a graded algebra with product induced by the shuffle product on the chain complex $C(A)$. In this section we recall the definition of the $r$-commutative differential forms $\Omega_{R}(A)$ and construct a natural homomorphism from them to $H^{R}(A)$.

First, let $\Omega_{u}(A)$ be the universal differential calculus over $A[4,14]$. This is a differential graded algebra with $\Omega_{u}^{0}(A)=A$ such that for any differential graded algebra $\Omega$ and any homomorphism $f: A \rightarrow \Omega^{0}$, there exists a unique differential graded algebra morphism $\tilde{f}$ for which the following diagram commutes:

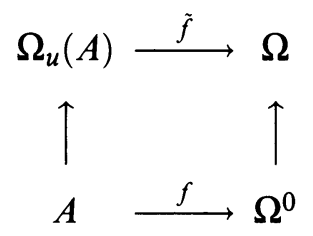

Let $m$ denote the multiplication map for $\Omega_{u}(A)$. We define the differential graded algebra of r-commutative differential forms over $A, \Omega_{R}(A)$, to be the quotient of $\Omega_{u}(A)$ by the differential ideal generated by $d l$ together with the range of the map

$$
m\left(\operatorname{id}_{A} \otimes d-\left(d \otimes \operatorname{id}_{A}\right) R\right): A \otimes A \rightarrow \Omega_{u}^{1}(A) .
$$

These have been treated before when $k$ is a field [1], and most of their properties carry over to the present situation. In particular, if $m$ now denotes the multiplication map for $\Omega_{R}(A)$, we have

$$
a d b=m\left(d \otimes \operatorname{id}_{A}\right) R(a \otimes b),
$$

hence

$$
d a d b=-m(d \otimes d) R(a \otimes b)
$$

for all $a, b \in A$, generalizing the usual identities which hold for differential forms:

$$
a d b=(d b) a, \quad d a d b=-d b d a .
$$

Theorem 3. Let $A$ be a strong $r$-commutative $r$-algebra. Then there is a unique graded algebra hommomorphism $f: \Omega_{R}(A) \rightarrow H^{R}(A)$,

$$
f(a)=[a], \quad f(d a)=[1 \otimes a] .
$$

Here [a] denotes the class in $H_{0}^{R}(A)$ of a regarded as an element of $C_{0}(A) \cong$ $A$, and $[1 \otimes a]$ denotes the class in $H_{1}^{R}(A)$ of a regarded as an element of $C_{1}(A) \cong A^{\otimes 2}$.

Proof. First, note that $C_{n}(A)=A \otimes_{A^{e}} A^{e} \otimes A^{\otimes n}$ is isomorphic to $A^{\otimes(n+1)}$ under the map

$$
a \otimes(b \otimes c) \otimes x \mapsto \rho(a \otimes(b \otimes c)) \otimes x,
$$

where $a, b, c \in A$ and $x \in A^{\otimes n}$. (An inverse is given by the map $a \otimes x \mapsto$ $a \otimes(1 \otimes 1) \otimes x$.$) In what follows we will identify C_{n}(A)$ with $A^{\otimes(n+1)}$ under this isomorphism. Note in particular that the boundary of $a \otimes b \otimes c \in C_{2}(A)$ is

$$
a b \otimes c-a \otimes b c+a c^{i} \otimes b_{i},
$$


where for any $b, c \in A$ we write $R(b \otimes c)=\sum_{i} c^{i} \otimes b_{i}$, and suppress explicit mention of the summation by the Einstein summation convention.

Next, note that $\Omega_{R}(A)$ may be defined as the graded algebra over $A=\Omega_{R}^{0}(A)$ generated by elements $d a$ for $a \in A$ subject to the relations:

$$
\begin{gathered}
d(\alpha a+b)=\alpha d a+d b, \quad d(a b)=a d b+(d b) a, \quad d 1=0, \\
a d b=m\left(d \otimes \operatorname{id}_{A}\right) R(a \otimes b), \quad d a d b=-m(d \otimes d) R(a \otimes b),
\end{gathered}
$$

for $a, b \in A, \alpha \in k$. (The first two relations serve to define $\Omega_{u}(A)$, and the last three define $\Omega_{R}(A)$ as a quotient of the universal differential calculus.) Thus $f: \Omega_{R}(A) \rightarrow H^{R}(A)$ is clearly unique if it exists, and existence reduces to showing that

$$
\begin{gathered}
{[1 \otimes(\alpha a+b)]=\alpha[1 \otimes a]+[1 \otimes b],} \\
{[1 \otimes a b]=[a][1 \otimes b]+[1 \otimes a][b],} \\
{[1 \otimes 1]=0,} \\
{[a][1 \otimes b]=\left[1 \otimes b^{i}\right]\left[a_{i}\right],} \\
{[1 \otimes a][1 \otimes b]=-\left[1 \otimes b^{i}\right]\left[1 \otimes a_{i}\right] .}
\end{gathered}
$$

Equation (9) is trivial. For equation (10), note that

$$
a(1 \otimes b)+(1 \otimes a) b-1 \otimes a b=a \otimes b+b^{i} \otimes a_{i}-1 \otimes a b
$$

in $C_{1}(A)$ is the boundary of $1 \otimes a \otimes b \in C_{2}(A)$. Similarly, the boundary of $1 \otimes 1 \otimes 1 \in C_{2}(A)$ is $1 \otimes 1$, proving equation (11). Equation (12) holds because $\left(1 \otimes b^{i}\right) a_{i}=a \otimes b=a(1 \otimes b)$ in $C_{1}(A)$. Equation (13) also holds at the level of chains:

$$
(1 \otimes a)(1 \otimes b)=1 \otimes a \otimes b-1 \otimes b^{i} \otimes a_{i}=-\left(1 \otimes b^{i}\right)\left(1 \otimes a_{i}\right) .
$$

In fact, the homomorphism $f: \Omega_{R}(A) \rightarrow H^{R}(A)$ is an isomorphism in degrees 0 and 1 . Given an $r$-algebra let $[A, A]_{R}$ denote the r-commutator, the span in $A$ of elements of the form $m\left(\mathrm{id}_{A \otimes A}-R\right)(a \otimes b)$.

Corollary 4. Let $A$ be an r-algebra. Then $H_{0}^{R}(A) \cong A /[A, A]_{R}$. If $A$ is strong and r-commutative then $f$ is an isomorphism from $\Omega_{R}^{0}(A)=A$ to $H_{0}^{R}(A)$, and from $\Omega_{R}^{1}(A)$ to $H_{1}^{R}(A)$.

Proof. Identifying $C_{n}(A)$ with $A^{\otimes(n+1)}$ as in the proof of Theorem 3, the cycles in $C_{0}(A)$ are identified with $A$, while the boundaries are identified with $[A, A]_{R}$. Thus when $A$ is strong and $r$-commutative, $f: \Omega_{R}^{0}(A) \rightarrow H_{0}^{R}(A)$ is an isomorphism.

Also, when $A$ is strong and $r$-commutative, we may define $g: C_{1}(A) \rightarrow$ $\Omega_{R}^{1}(A)$ by $g(a \otimes b)=a d b$. Since boundaries in $C_{1}(A)$ are spanned by elements of the form $a b \otimes c-a \otimes b c+a c^{i} \otimes b_{i}$, which are annihilated by $g, g$ serves to define an inverse to $f$.

It would be interesting to find smoothness conditions on $A$ that would imply that $f$ is an isomorphism in all degrees, thus generalizing the work of Hochschild, Kostant and Rosenberg [11].

\section{Conclusions}

One could proceed to develop the theory of braided Hochschild and (in the strong case) cyclic homology for $r$-algebras, but clearly it is more urgent to 
calculate it in certain cases and develop an understanding of its significance. The lack of "stability" under deformations of ordinary Hochschild homology is well-known. For noncommutative tori this was observed by Connes [4] (and see also [26]), while for quantum groups this was shown by Feng and Tsygan [8]. In both these cases the algebras in question are $r$-commutative, so it would be interesting to see if working with braided Hochschild homology would increase stability. We know $H_{i}^{R}(A)$ for $i=0,1$ for a variety of algebras including noncommutative tori and the Weyl algebra, by Corollary 4 and the results of [1], and in these cases stability is indeed enhanced.

Some of our constructions could be placed more firmly in a categorical framework by assuming that the category $\mathscr{V}$ admits direct sums, kernels and cokernels, and so on. Various extensions of the notion of a braided category appear in the literature, such as "rigid quasitensor categories" and "braided compact closed categories", so adopting such an approach would largely be a matter of choosing among the available definitions.

\section{REFERENCES}

1. J. Baez, R-commutative geometry and quantization of Poisson algebras, Adv. Math. 95 (1992), 61-91.

2. __ Differential calculi for quantum vector spaces with Hecke-type relations, Lett. Math. Phys. 23 (1991), 133-141.

3. H. Cartan and S. Eilenberg, Homological algebra, Princeton Univ. Press, Princeton, N.J., 1956.

4. A. Connes, Non-commutative differential geometry, Publ. Math. IHES 62 (1985), 257-360.

5. P. Deligne and J. Milne, Tannakian categories, Lecture Notes in Math., vol. 900, Springer, New York, 1982.

6. V. Drinfeld, Quantum groups, Proc. Internat Congr. Math., 1986, pp. 798-820.

7. L. Faddeev, N. Reshetikhin, and L. Takhtajan, Quantization of Lie groups and Lie algebras, Leningrad Math. J. 1 (1990), 193-225.

8. P. Feng and B. Tsygan, Hochschild and cyclic homology of quantum groups, Comm. Math. Phys. 140, 481-521.

9. J. Fröhlich and F. Gabbiani, Braid group statistics in local quantum theory, preprint.

10. P. Freyd and D. Yetter, Braided compact closed categories with applications to low dimensional topology, Adv. Math. 77 (1989), 156-182.

11. G. Hochschild, B. Kostant and A. Rosenberg, Differential forms on regular affine algebras, Trans. Amer. Math. Soc. 102 (1962), 383-408.

12. A. Joyal and R. Street, Braided monoidal categories, Mathematics Reports 86008, Macquarie University, 1986.

13. _ The geometry of tensor calculus I, Adv. Math. 88 (1991), 55-112.

14. M. Karoubi, Homologie cyclique et $K$ théorie, Astérisque 149 (1987), 1-147.

15. L. Kauffman, On knots, Princeton Univ. Press, Princeton, N.J., 1986.

16. K Knots and physics, World Scientific, New Jersey, 1991.

17. V. Lyubashenko, Hopf algebras and vector-symmetries, Uspehi Mat. Nauk 41 (1986), 185186.

18. S. Mac Lane, Natural associativity and commutativity, Rice Univ. Studies 69 (1963), 28-46.

19. S. Majid, Quasitriangular Hopf algebras and Yang-Baxter equations, Internat J. Modern Phys. A 5 (1990), 1-91.

20. Yu. Manin, Quantum groups and noncommutative geometry, Publ. du Centre de Récherches Math., Université de Montréal, Montréal, 1988. 
21. - Topics in noncommutative geometry, Princeton Univ. Press, Princeton, N.J., 1991.

22. W. Pusz, Twisted canonical anticommutation relations, Rep. Math. Phys. 27 (1989), 349360.

23. W. Pusz and S. Woronowicz, Twisted second quantization, Rep. Math. Phys. 27 (1989), 231-257.

24. J.-L. Loday and D. Quillen, Cyclic homology and the Lie algebra homology of matrices, Comment. Math. Helv. 59 (1984), 565-591.

25. N. Reshetikhin and V. Turaev, Ribbon graphs and their invariants derived from quantum groups, Comm. Math. Phys. 127 (1990), 1-26.

26. M. Rieffel, Noncommutative tori, a case study of noncommutative differential manifolds, Contemp. Math. 105 (1990), 191-211.

27. N. Saavedra Rivano, Catégories Tannakiennes, Lecture Notes in Math., vol. 265, Springer, Berlin-Heidelberg, 1975.

28. P. Seibt, Cyclic homology of algebras, World Scientific, Singapore, 1987.

29. B. Tsygan, Homology of matrix Lie algebras over rings and Hochschild homology, Uspehi Mat. Nauk 38 (1983), 217-218.

30. K.-H. Ulbrich, On Hopf algebras and rigid monoidal categories, Israel J. Math. 72 (1990), 252-256.

31. J. Wess and B. Zumino, Covariant differential calculus on the quantum hyperplane, preprint.

32. C. Yang and M. Ge, Braid group, knot theory, and statistical mechanics, World Scientific, 1989.

33. B. Zumino, Deformation of the quantum mechanical phase space with bosonic or fermionic coordinates, preprint.

Department of Mathematics, University of California, Riverside, California 92521 